УДК 538.951, 539.3, 539.5

DOI: $10.17308 / \mathrm{kcmf} .2019 .21 / 719$

Поступила в редакцию 13.01.2019

Подписана в печать 15.02.2019

\title{
ОСОБЕННОСТИ РЕЛАКСАЦИИ СДВИГОВОЙ УПРУГОСТИ МЕТАЛЛИЧЕСКИХ СТЕКОЛ
}

\author{
(C) 2019 Ю. П. Митрофанов* \\ Воронежский государственный педагогический университет \\ ул. Ленина, 86, 394043 Воронеж, Российская Федерация
}

\begin{abstract}
Аннотация. Работа направлена на установление закономерностей изменения сдвиговой упругости, возникающих при структурной релаксации металлических стекол на основе $\mathrm{Pd}$ и $\mathrm{Zr}$. Измерения модуля сдвига выполнялись на частотах около 500 кГц. Несмотря на отличия в физических свойствах исследованных металлических стекол (химический состав, стеклообразующая способность, температуры стеклования и др.), наблюдаются определенные общие закономерности релаксации их сдвиговой упругости при термообработке.
\end{abstract}

Ключевые слова: металлические стекла, сдвиговая упругость, релаксация.

\section{ВВЕДЕНИЕ}

Мгновенный (нерелаксированный) модуль сдвига $G_{\infty}$ считается важным термодинамическим параметром некристаллических веществ [1]. Экспериментально показано, например, что свободная энергия активации $\Delta F$ атомных перестроек в органических и металлических переохлажденных жидкостях пропорциональна величине $G_{\infty}[2,3]$. Это соотношение означает наличие взаимосвязи между быстрой атомно-молекулярной динамикой некристаллической структуры и медленной релаксацией ее физических свойств. В рамках такого подхода величина и изменение мгновенных модулей упругости вещества является индикатором особенностей релаксации некристаллической среды. Более того, определяющая роль принадлежит не дилатационной, а сдвиговой компоненте, т.е. мгновенному модулю сдвига [1].

Вопрос о природе сдвиговой доминанты остается открытым и является предметом многочисленных дискуссий [1]. Однако модели, описывающие механизм взаимосвязи между $\Delta F$ и $G_{\infty}$, дают схожие выводы. Их можно сформулировать следующим образом [1]: 1) энергия активации атомных структурных перестроек в основном представляет собой работу, которую необходимо совершить группе атомов, чтобы эта перестройка произошла; 2) совершаемая работа зависит от упругих свойств окружающей среды, характеризуемой мгновенны-

Митрофанов Юрий Петрович, e-mail: mitrofanovyup@gmail.com ми модулями упругости; 3) атомные перестройки представляют собой чистую сдвиговую деформацию, т.е. дилатационный вклад в общий баланс упругой энергии является несущественным, или, другими словами, мгновенный модуль сдвига контролирует активационный процесс.

Безотносительно к интерпретации природы соотношения между $\Delta F$ и $G_{\infty}$ существует актуальная задача по накоплению и обобщению экспериментальных данных об изменении модуля сдвига $G_{\infty}$ в различных температурных условиях для некристаллических веществ разного химического состава. Особенный интерес вызывает интервал температур, в котором происходит интенсивная структурная релаксация и/или стеклование. Следует подчеркнуть, что явление структурной релаксации в металлических стеклах является масштабным, поскольку сильно изменяет их физические свойства [4]. Природа структурной релаксации металлических стекол остается предметом дискуссий. Отсутствие адекватной физической модели структурной релаксации в известной степени сдерживает технологическое применение этих материалов, поскольку предсказание их поведения в различных термомеханических условиях в настоящее время не представляется возможным.

Измерение модуля сдвига $G_{\infty}$ предполагает применение акустических методов на частотах, при которых время измерения много меньше времени релаксации структуры. В эксперименте таким условиям отвечают частоты 0.5-1 MHz и выше. Экспери- 
ментальная реализация таких измерений в металлических стеклах вызывает значительные сложности. С одной стороны, измерения модуля сдвига $G_{\infty}$ необходимо выполнять непосредственно в процессе термообработки при температурах, которые значительно больше комнатной. При этом возникает принципиальная трудность создания надежного акустического контакта между исследуемым веществом и источником (приемником) акустических волн. С другой стороны, измерения $G_{\infty}$ необходимо проводить с прецизионной точностью. Последнее требование является необходимым условием для надежной идентификации тонких эффектов релаксации некристаллической металлической структуры. Среди многих ультразвуковых методов вышеперечисленным требованиям удовлетворяет метод электромагнитного акустического резонанса (ЭМАР) [5], в основе которого лежит явление электромагнитного акустического преобразования [6].

В настоящей работе была поставлена цель выполнить экспериментальное исследование изменения модуля сдвига $G_{\infty}$ металлических стекол разного химического состава при их термообработке. Измерения $G_{\infty}$ были выполнены на частотах около $500 \mathrm{kHz}$ методом ЭМАР. В результате получены экспериментальные данные об изменении величины $G_{\infty}$ в условиях линейного нагрева, что позволило установить общие закономерности релаксации модуля $G_{\infty}$ ниже и выше калориметрической температуры стеклования $T_{g}$.

\section{ЭКСПЕРИМЕНТАЛЬНАЯ ЧАСТЬ}

Экспериментальные исследования были выполнены на металлических стеклах на основе $\mathrm{Pd}$ и $\mathrm{Zr}: \mathrm{Pd}_{43.2} \mathrm{Cu}_{28} \mathrm{Ni}_{8.8} \mathrm{P}_{20}, \mathrm{Pd}_{41.25} \mathrm{Cu}_{41.25} \mathrm{P}_{17.5}, \mathrm{Zr}_{46} \mathrm{Cu}_{46} \mathrm{Al}_{8}$ и $\mathrm{Zr}_{46} \mathrm{Cu}_{45} \mathrm{Al}_{7} \mathrm{Ti}_{2}$ (at. \%). Кристаллические сплавы, которые использовались для приготовления стекол, были получены путем сплавления соответствующих химических компонентов. Образцы металлических стекол были получены методами реактивной закалки или вакуумного всасывания расплава. Скорость закалки расплава составляла около $200 \mathrm{~K} / \mathrm{s}$. Полученные отливки имели размеры $(2 \div 2.5) \times 5 \times(60 \div 65) \mathrm{mm}^{3}$. Из них вырезались образцы размером $(2 \div 2.5) \times 5 \times 5 \mathrm{~mm}^{3}$ для измерений модуля сдвига. Структурное состояние образцов до и после термообработки контролировалось с помощью рентгеноструктурного анализа.

Исследования релаксации $G_{\infty}$ были выполнены на частотах около $500 \mathrm{kHz}$ с помощью специальной экспериментальной установки, разработанной автором (далее, обозначим $G_{\infty}$ просто как $G$ ). Фи- зическую основу этой установки составляет метод ЭМАР [4]. В этом методе возбуждение упругих волн в образце происходит за счет взаимодействия внешнего магнитного поля и переменного электрического тока, текущего по поверхности образца. Метод ЭМАР обладает принципиально важным достоинством - отсутствием прямого акустического контакта между образцом и источником/приемником волн. Это позволяет выполнять измерения упругих модулей непосредственно в процессе термообработки (т.е. in situ), причем до весьма высоких температур (1000 K).

Релаксация модуля сдвига в результате термообработки характеризовалась величиной $g=G / G_{r t}-1 \approx f^{2} / f_{r t}{ }^{2}-1$, где $g$ - относительное изменение модуля сдвига, $G_{r t}$ - значение модуля сдвига исходного стекла при комнатной температуре, $f$ - текущая резонансная частота поперечных колебаний и $f_{r t}-$ значение этой частоты для исходного образца при комнатной температуре. Относительная точность измерения $g$ вблизи комнатной температуры составляла $0.005 \%$. С ростом температуры точность измерений падает вследствие увеличения внутреннего трения материала, достигая $\approx 0.02 \%$ при температурах $700 \mathrm{~K}-750 \mathrm{~K}$. Измерения $g$ были выполнены при скорости нагрева $3 \mathrm{~K} / \mathrm{min}$ и давлении около $0.1 \mathrm{~Pa}$. Температурная зависимость модуля сдвига $G$ определялась по формуле $G(T)=G_{r t}(1+g(T))$, где значение модуля сдвига при комнатной температуре $\left(G_{r t}\right)$ было взято из экспериментальных данных, полученных в работах [7-10]. Температура стеклования $T_{g}$ определялась методом дифференциальной сканирующей калориметрии (ДСК) на той же скорости нагрева с помощью прибора Hitachi DSC 7020.

\section{РЕЗУЛЬТАТЫ И ИХ ОБСУЖДЕНИЕ}

На рис. 1 представлены результаты измерения температурной зависимости модуля сдвига исследуемых металлических стекол для исходного («run1») и релаксированного («run2») состояний. Схема термообработки образцов состояла в следующем. Образец исходного стекла нагревался со скоростью $3 \mathrm{~K} / \mathrm{min}$ до некоторой температуры $T_{a}>T_{g}$ («run1») и далее охлаждался с этой же скоростью до комнатной температуры $T_{r t}$ В результате при $T_{r t}$ получалось релаксированное стеклообразное состояние. После этого дважды выполнялась термообработка образца по той же самой схеме («run2» и «run3» соответственно).

Видно, что в исходном стеклообразном состоянии модуль сдвига $\left(G_{i n}\right)$ во всех случаях монотонно 

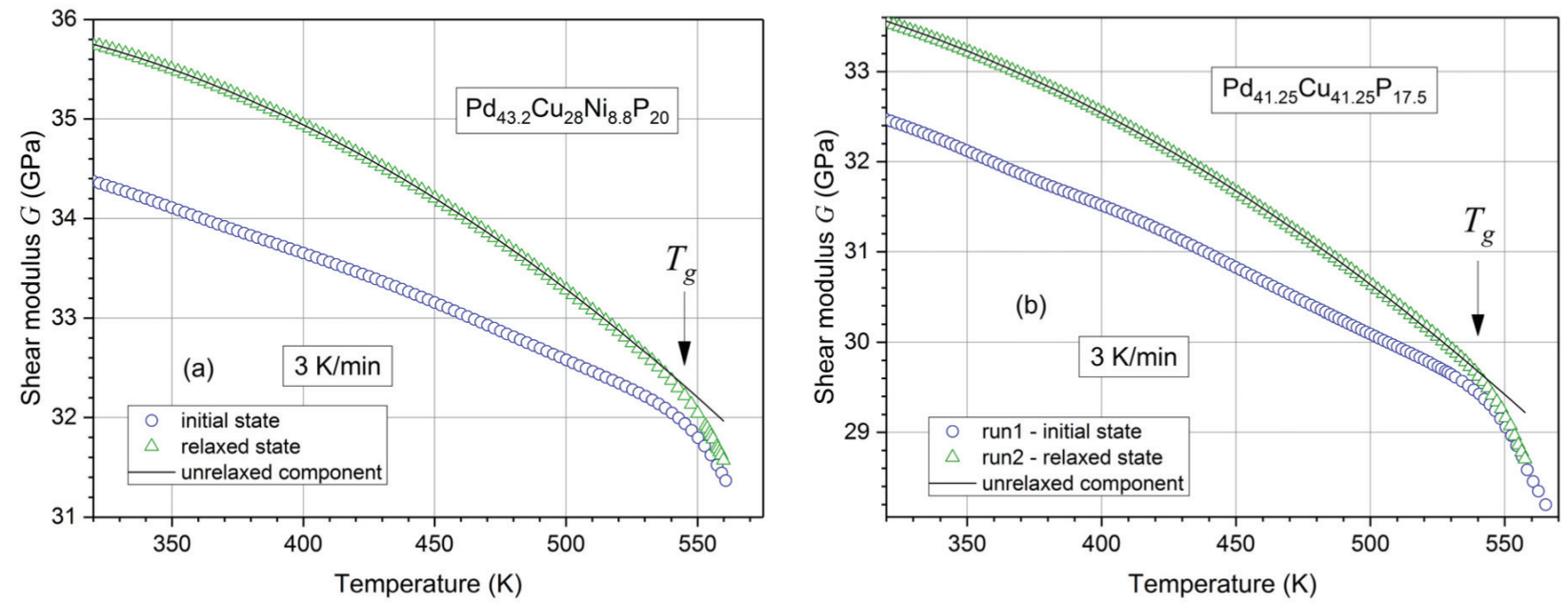

$a$

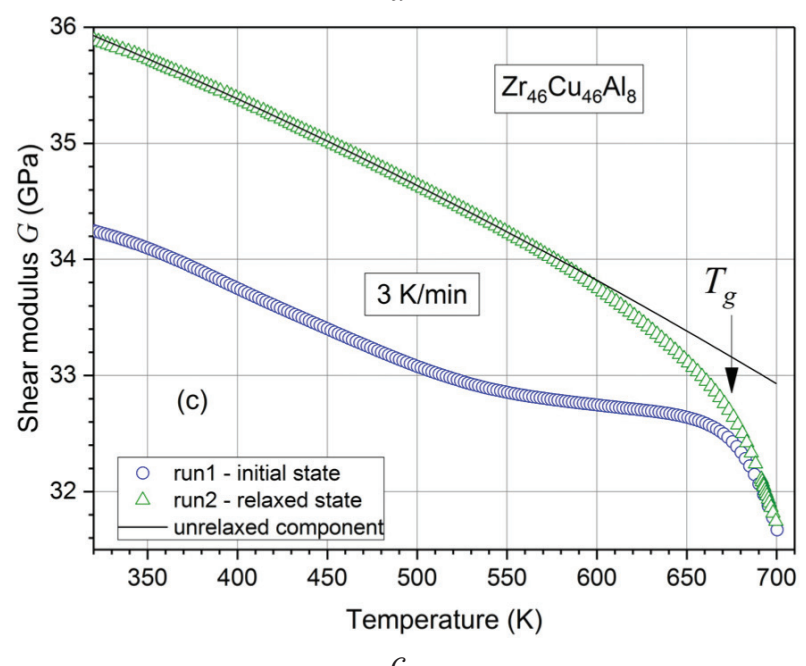

$b$

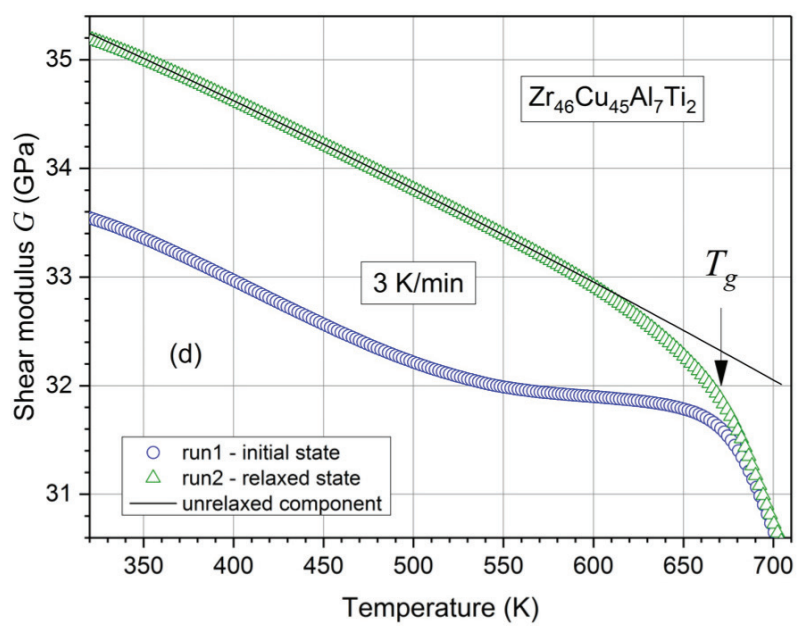

$d$

Рис. 1. Температурные зависимости модуля сдвига металлических стекол $\mathrm{Pd}_{43.2} \mathrm{Cu}_{28} \mathrm{Ni}_{8.8} \mathrm{P}_{20}(a), \mathrm{Pd}_{41.25} \mathrm{Cu}_{41.25} \mathrm{P}_{17.5}$ $(b), \mathrm{Zr}_{46} \mathrm{Cu}_{45} \mathrm{Al}_{7} \mathrm{Ti}_{2}(c)$ и $\mathrm{Zr}_{46} \mathrm{Cu}_{46} \mathrm{Al}_{8}(d)$ в исходном и релаксированном состояниях. Нерелаксационная компонента $G_{u r}$ представлена сплошной линией

[Fig. 1. Temperature dependences of the shear modulus of glassy $\mathrm{Pd}_{43.2} \mathrm{Cu}_{28} \mathrm{Ni}_{8.8} \mathrm{P}_{20}$ (a), $\mathrm{Pd}_{41.25} \mathrm{Cu}_{41.25} \mathrm{P}_{17.5}$ (b), Zr${ }_{46} \mathrm{Cu}_{45} \mathrm{Al}_{7} \mathrm{Ti}_{2}(\mathrm{c})$ and $\mathrm{Zr}_{46} \mathrm{Cu}_{46} \mathrm{Al}_{8}(\mathrm{~d})$ in the initial and relaxed states. A solid line shows the unrelaxed component $G_{u r}$ ]

снижается с температурой («run1»). Вблизи калориметрической температуры стеклования $T_{g}$ происходит быстрое увеличение наклона кривой $G(T)$. Состояние переохлажденной жидкости (т. е. при температурах $T>T_{g}$ ) характеризуется относительно быстрым снижением модуля сдвига в сравнении со стеклообразным состоянием. При этом происходит быстрый (экспоненциальный) рост внутреннего трения с температурой. Последнее обстоятельство является причиной снижения точности измерений так, что при некоторой температуре $T_{a}$ они становятся невозможными. Вышеописанное поведение $G_{i n i}(T)$ не зависит от химического состава исследованных стекол.

После охлаждения образца от $T_{a}$ до $T_{r t}$ получается релаксированное стекло, модуль сдвига ко- торого $\left(G_{r e l}\right)$ примерно на 3-5 \% выше исходного значения (см. табл. 1). Характер изменения модуля сдвига релаксированного стекла с температурой («run2») в целом такой же, как и для исходного состояния: величина $G_{r e l}$ снижается с температурой как ниже, так и выше $T_{g}$. При 3-м нагреве («run3») температурная зависимость модуля сдвига оказывается идентична предыдущей зависимости $G_{r e l}(T)$ (в связи с этим 3-е измерение, «run 3», на рис. 1 не показано). Разница $\Delta G_{s r}=G_{r e l}-G_{i n i}$ характеризует изменение модуля сдвига металлических стекол в результате структурной релаксации. Величина $\Delta G_{s r}$ является положительной вне зависимости от химического состава исследованных стекол. По мере приближения системы к температуре $T_{g}$ величина $\Delta G_{s r}$ стремится к нулю. 
Табл. 1. Параметры сдвиговой упругости металлических стекол $\mathrm{Pd}_{43.2} \mathrm{Cu}_{28} \mathrm{Ni}_{8.8} \mathrm{P}_{20}, \mathrm{Pd}_{41.25} \mathrm{Cu}_{41.25} \mathrm{P}_{17.5}, \mathrm{Zr}_{46} \mathrm{Cu}_{45} \mathrm{Al}_{7} \mathrm{Ti}_{2}$ и $\mathrm{Zr}_{46} \mathrm{Cu}_{46} \mathrm{Al}_{8}: G_{r t}$ - модуль сдвига исходного стекла при комнатной температуре $T_{r t} ; \Delta G_{s r} / G_{r t}-$ относительное изменение модуля сдвига в результате структурной релаксации, взятое при $T_{r t} ; G_{0 K}, \alpha_{1}$ и $\alpha_{2}-$ параметры аппроксимации температурной зависимости модуля сдвига в релаксированном состоянии, где $G_{0 K}-$ модуль сдвига при $0 \mathrm{~K}, \alpha_{1}$ и $\alpha_{2}$ - параметры, характеризующие ангармонический и электронные вклады в модуль сдвига; $G_{r}^{r t}$ - величина релаксационного вклада при $T_{r t}$

[Table 1. Parameters of the shear elasticity of metallic glassy $\mathrm{Pd}_{43.2} \mathrm{Cu}_{28} \mathrm{Ni}_{8.8} \mathrm{P}_{20}, \mathrm{Pd}_{41.25} \mathrm{Cu}_{41.25} \mathrm{P}_{17.5}, \mathrm{Zr}_{46} \mathrm{Cu}_{45} \mathrm{Al}_{7} \mathrm{Ti}_{2}$ and $\mathrm{Zr}_{46} \mathrm{Cu}_{46} \mathrm{Al}_{8}: G_{r t}$ is the shear modulus of the initial glass at room temperature $T_{r t} ; \Delta G_{s t} / G_{r t}$ is the relative change of the shear modulus due to structural relaxation measured at $T_{r t} ; G_{0 K}, \alpha_{1}$ and $\alpha_{2}$ are the fitting parameters for the temperature dependencies of the shear modulus in the relaxed glassy state, where $G_{0 K}$ is the shear modulus of the initial glassy state at $0 \mathrm{~K}, \alpha_{1}$ and $\alpha_{2}$ are the parameters characterizing the anharmonic and electronic contributions to the shear modulus; $G_{r}^{r t}$ is the relaxation contribution taken at $T_{r t}$ ]

\begin{tabular}{|c|c|c|c|c|c|c|c|}
\hline № & $\begin{array}{c}\text { Alloy } \\
\text { (at. \%) }\end{array}$ & $\begin{array}{c}G_{r t} \\
(\mathrm{GPa})\end{array}$ & $\begin{array}{c}\Delta G_{s r} / G_{r t} \\
(\%)\end{array}$ & $\begin{array}{c}G_{0 K} \\
(\mathrm{GPa})\end{array}$ & $\begin{array}{c}\alpha_{1} \\
\left(10^{-4} \mathrm{~K}^{-1}\right)\end{array}$ & $\begin{array}{c}\alpha_{2} \\
\left(10^{-8} \mathrm{~K}^{-2}\right)\end{array}$ & $\begin{array}{c}G_{r}^{r t} \\
(\mathrm{GPa})\end{array}$ \\
\hline 1 & $\mathrm{Pd}_{43.2} \mathrm{Cu}_{28} \mathrm{Ni}_{8.8} \mathrm{P}_{20}$ & $34.50[8]$ & 3.97 & 34.47 & 4.442 & -102.512 & -1.37 \\
\hline 2 & $\mathrm{Pd}_{41.25} \mathrm{Cu}_{41.25} \mathrm{P}_{17.5}$ & $32.70[7]$ & 3.21 & 33.16 & 3.757 & -105.562 & -1.05 \\
\hline 3 & $\mathrm{Zr}_{46} \mathrm{Cu}_{46} \mathrm{Al}_{8}$ & $34.30[7]$ & 4.83 & 37.64 & -1.111 & -9.663 & -1.66 \\
\hline 4 & $\mathrm{Zr}_{46} \mathrm{Cu}_{45} \mathrm{Al}_{7} \mathrm{Ti}_{2}$ & $33.61[9]$ & 4.92 & 37.43 & -1.633 & -5.958 & -1.65 \\
\hline
\end{tabular}

Рассмотрим особенности изменения модуля сдвига с ростом температуры для $T<T_{g}$. Для этого была вычислена первая производная модуля сдвига по температуре $d G / d T$ (рис. 2). Установлено, что, вне зависимости от химического состава стекла и его структурного состояния, величина $d G /$ $d T$ ниже $T_{g}$ медленно изменяется с ростом $T$. При этом выше некоторой характеристической температуры $T_{s r}$ значение $d G / d T$ зависит от структурного состояния стекла. Так, для стекол на основе $\mathrm{Pd}$ и $\mathrm{Zr}$ при температурах больших $\approx 360 \mathrm{~K}$ и $\approx 450 \mathrm{~K}$, соответственно, величина $d G / d T$ в релаксированном состоянии всегда меньше, чем в исходном. Из рис. 2 также следует, что в исходных стеклах на основе $\mathrm{Zr}$ величина модуля сдвига снижается линейно вплоть до $T_{s r}$, поскольку выполняется условие $d G / d T=$ const. Выше $T_{s r}$ функция $G(T)$ становится нелинейной. Для металлических стекол на основе $\operatorname{Pd}$ зависимость $G(T)$ нелинейна во всем диапазоне температур $T<T_{g}$, т. к. величина $d G / d T=f(T)$. Известно, что для металлических стекол процесс интенсивной («быстрой») структурной релаксации наблюдается выше 350-400 K [11]. Следовательно, изменение величины $G$ с температурой при $T<T_{s r}$ обусловлено ангармонизмом атомных колебаний, а в интервале $T_{s r}<T<T_{g}$, кроме ангармонической компоненты, существует и релаксационная.

Вблизи и выше $T_{g}$ происходит быстрое изменение величины $d G / d T$ : по абсолютному значению она растет примерно в 3-4 раза (в зависимости от химического состава стекла). При температурах $T>T_{g}+25 \mathrm{~K}$ величина $d G / d T$ перестает изменяться с температурой. Таким образом, стеклование со- провождается значительным изменением характера поведения сдвиговой упругости некристаллической металлической структуры.

Чтобы выделить ангармонический и релаксационный вклады, был выполнен анализ температурных зависимостей модуля сдвига релаксированных стекол. В релаксированном состоянии характер зависимости $G(T)$ отличается от исходного. Для стекол на основе $\mathrm{Zr}$ при $T \ll T_{g}$ величина $d G / d T=$ const, т. е. модуль сдвига $G$ линейно снижается с температурой. Для стекол на основе Pd величина $d G / d T$ ниже $T_{g}$ является линейной функцией температуры (см. рис. 2). Таким образом, низкотемпературную часть зависимости $G(T)$ релаксированных стекол можно аппроксимировать полиномом второй степени: $G(T)=G_{0 K}\left(1+\alpha_{1} T+\alpha_{2} T^{2}\right)$, где $G_{0 K}-$ модуль сдвига при $0 \mathrm{~K}, \alpha_{1}$ и $\alpha_{2}-$ константы. Такое же уравнение используется для анализа кристаллического состояния $[12,13]$. Оно показывает, что если кристалл свободен от точечных дефектов, то вторая производная модуля сдвига не должна зависеть от температуры, т. е. должна быть константой. При этом первый коэффициент, $\alpha_{1,}$ несет информацию об ангармонизме межатомного потенциала, а второй, $\alpha_{2}$, характеризует вклад свободных электронов $[12,13]$. В рамках этого подхода был выполнен дальнейший анализ кривых $G(T)$.

На вставках к рис. 2 показаны температурные зависимости второй производной модуля сдвига по температуре $d^{2} G / d T^{2}$ для релаксированного состояния. Видно, что для стекол на основе $\mathrm{Pd}$ значение $d^{2} G / d T^{2}=$ const ниже $\approx 500 \mathrm{~K}$, а для циркониевых стекол - ниже $\approx 550 \mathrm{~K}$. Поскольку температу- 

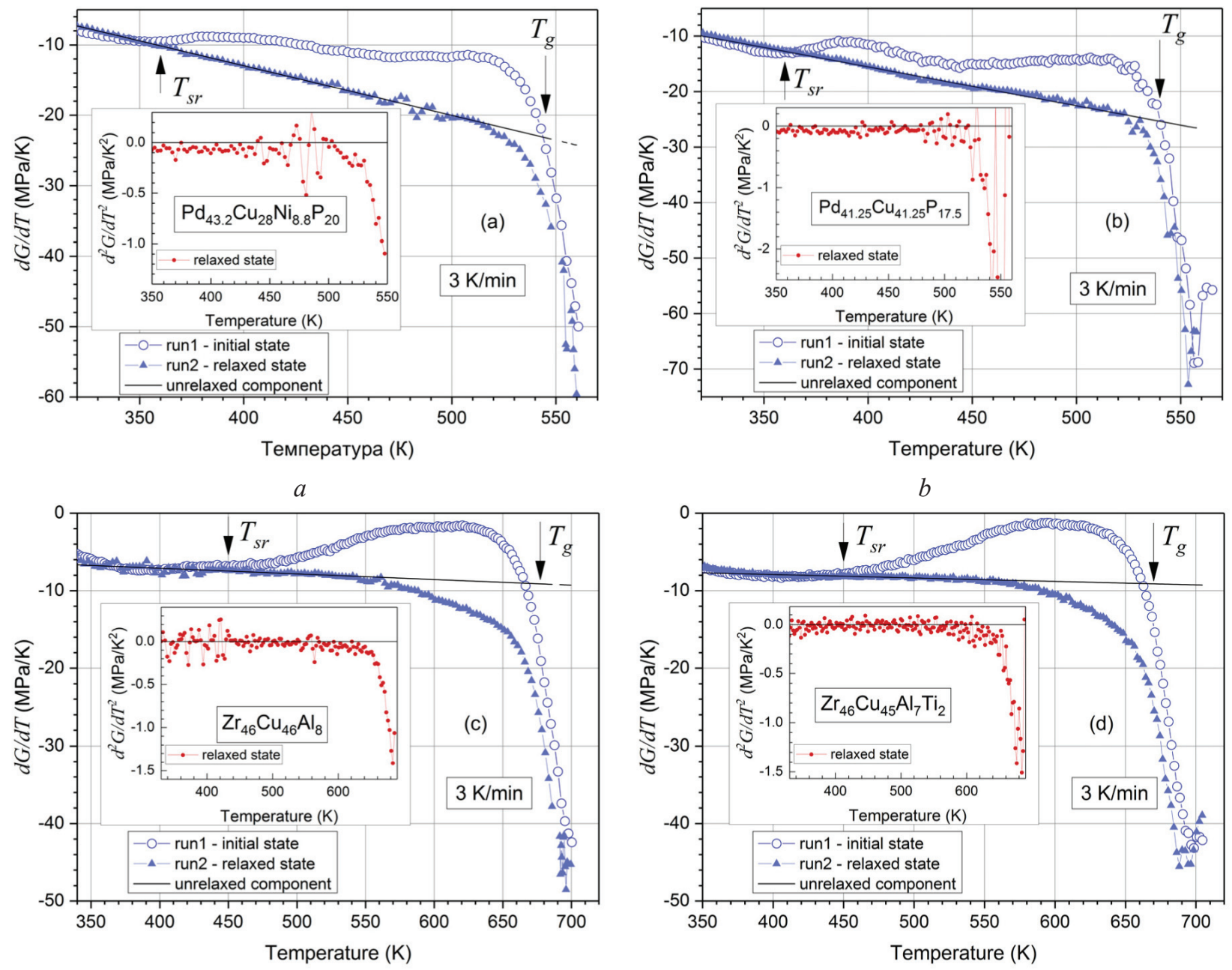

$d$

Рис. 2. Температурная зависимость первой производной модуля сдвига по температуре $(d G / d T)$ для металлических стекол $\mathrm{Pd}_{43.2} \mathrm{Cu}_{28} \mathrm{Ni}_{8.8} \mathrm{P}_{20}(a), \mathrm{Pd}_{41.25} \mathrm{Cu}_{41.25} \mathrm{P}_{17.5}(b), \mathrm{Zr}_{46} \mathrm{Cu}_{45} \mathrm{Al}_{7} \mathrm{Ti}_{2}(c)$ и $\mathrm{Zr}_{46} \mathrm{Cu}_{46} \mathrm{Al}_{8}(d)$. На вставках показана температурная зависимость второй производной модуля сдвига по температуре $\left(d^{2} G / d T^{2}\right)$. Нерелаксационная компонента $\left(d G_{u r} / d T\right)$ представлена сплошной линией

[Fig. 2. Temperature dependence of the first temperature derivative of the shear modulus $(d G / d T)$ of glassy $\mathrm{Pd}_{43.2} \mathrm{Cu}_{28} \mathrm{Ni}_{8.8} \mathrm{P}_{20}$ (a), $\mathrm{Pd}_{41.25} \mathrm{Cu}_{41.25} \mathrm{P}_{17.5}$ (b), $\mathrm{Zr}_{46} \mathrm{Cu}_{45} \mathrm{Al}_{7} \mathrm{Ti}_{2}$ (c) and $\mathrm{Zr}_{46} \mathrm{Cu}_{46} \mathrm{Al}_{8}$ (d). The insets show temperature dependence of the second derivative of the shear modulus $\left(d^{2} G / d T^{2}\right)$. A solid line corresponds to the unrelaxed component $\left.d G_{u r} / d T\right]$

ра Дебая металлических стекол на основе $\mathrm{Pd}$ и $\mathrm{Zr}$ не превышает 350 K [7], аппроксимация данных $G_{r e l}(T)$ с помощью вышеуказанного уравнения для указанных металлических стекол была выполнена в интервалах соответственно 350-500 K и 350-550 К. Результат аппроксимации позволяет определить вклад $G_{u r}(T)$, который характеризует изменение модуля сдвига, не связанное со структурной релаксацией (см. значения параметров $\alpha_{1}$ и $\alpha_{2}$ в табл. 1). Далее путем вычитания компоненты $G_{u r}(T)$ из зависимости $G(T)$ были получены температурные зависимости релаксационного вклада $G_{r}(T)$ (см. рис. 3). Установлено, что в исходном стекле вели- чина $G_{r}$ растет ниже $T_{g}$, а выше $T_{g}$ она относительно быстро снижается с температурой. В релаксированном стекле вклад $G_{r}$ является ненулевым вблизи и выше $T_{g}$, где он снижается так же, как и в исходном состоянии.

Исследованные металлические стекла обладают весьма необычными физическими свойствами, которые выделяют их среди других составов. Так, расплав $\mathrm{Pd}_{43.2} \mathrm{Cu}_{28} \mathrm{Ni}_{8.8} \mathrm{P}_{20}$ является лучшим металлическим стеклообразователем, поскольку его критическая скорость охлаждения составляет всего $0.3 \mathrm{~K} /$ $\min [14]$. Стекло $\mathrm{Pd}_{41.25} \mathrm{Cu}_{41.25} \mathrm{P}_{17.5}$ является уникальным в силу того, что его кристаллизация происхо- 

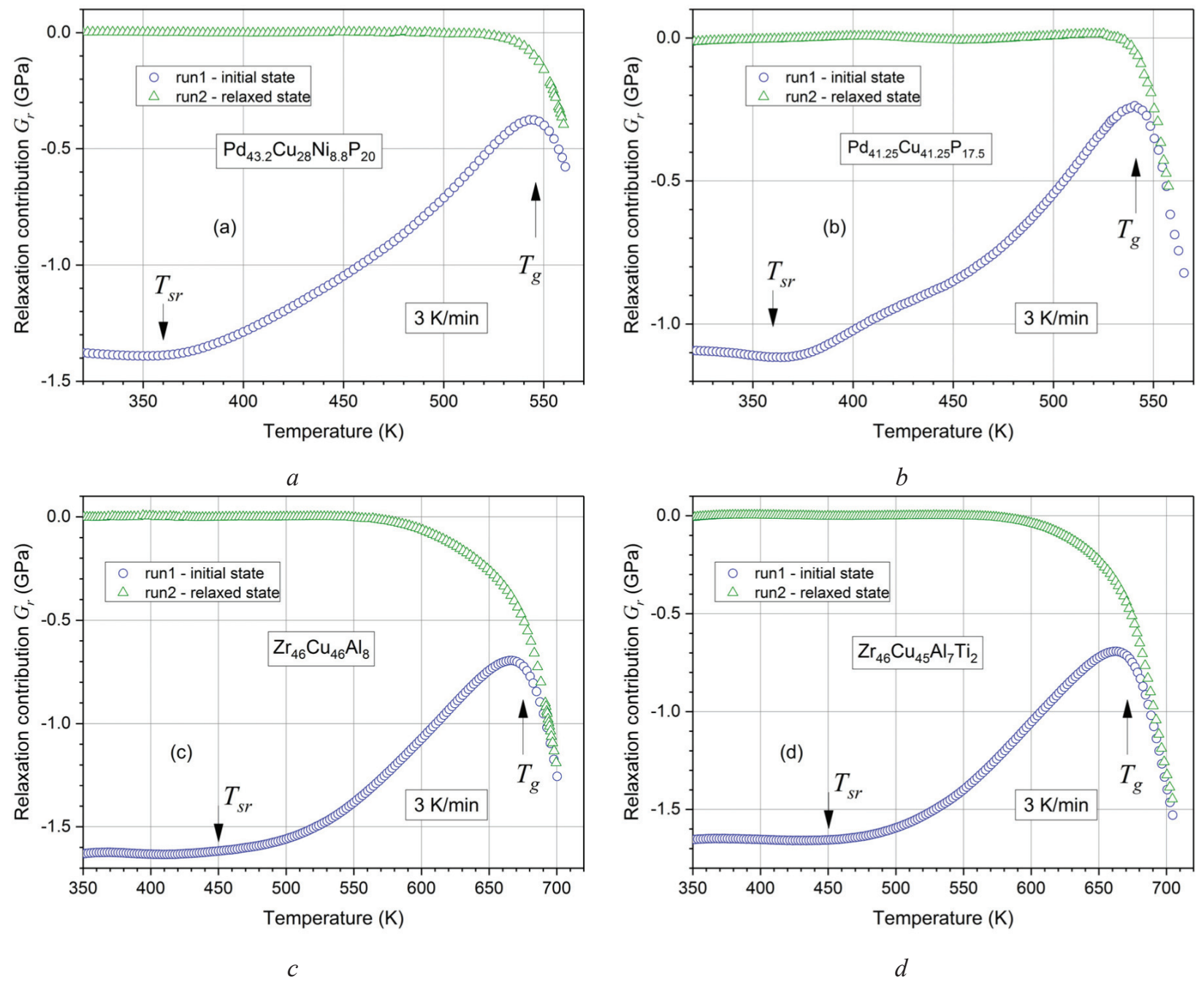

Рис. 3. Температурная зависимость релаксационного вклада $G_{r}$ для металлических стекол $\mathrm{Pd}_{43.2} \mathrm{Cu}_{28} \mathrm{Ni}_{8.8} \mathrm{P}_{20}(a)$, $\mathrm{Pd}_{41.25} \mathrm{Cu}_{41.25} \mathrm{P}_{17.5}(b), \mathrm{Zr}_{46} \mathrm{Cu}_{45} \mathrm{Al}_{7} \mathrm{Ti}_{2}(c)$ и $\mathrm{Zr}_{46} \mathrm{Cu}_{46} \mathrm{Al}_{8}(d)$ в исходном и релаксированном состояниях

[Fig. 3. Temperature dependence of the relaxation contribution $G_{r}$ of glassy $\mathrm{Pd}_{43.2} \mathrm{Cu}_{28} \mathrm{Ni}_{8.8} \mathrm{P}_{20}(a), \mathrm{Pd}_{41.25} \mathrm{Cu}_{41.25} \mathrm{P}_{17.5}($ b) , $\mathrm{Zr}_{46} \mathrm{Cu}_{45} \mathrm{Al}_{7} \mathrm{Ti}_{2}(c)$ and $\mathrm{Zr}_{46} \mathrm{Cu}_{46} \mathrm{Al}_{8}(d)$ in the initial and relaxed states]

дит полиморфно в единственную тетрагональную фазу $\mathrm{Pd}_{2} \mathrm{Cu}_{2} \mathrm{P}$ и его плотность при этом уменьшается [15]. Металлическое стекло $\mathrm{Zr}_{46} \mathrm{Cu}_{45} \mathrm{Al}_{7} \mathrm{Ti}_{2}$ имеет высокую пластичность при комнатной температуре (около 30 \%), в то время как стекло $\mathrm{Zr}_{46} \mathrm{Cu}_{46} \mathrm{Al}_{8}$ таким свойством не обладает [9]. Как видно из рис. 1 и 2 стекла отличаются также величиной температуры стеклования $T_{g}$.

Таким образом, несмотря на разный химический состав или разные физические свойства, характер зависимости $G_{r}(T)$ является аналогичнылм для всех исследованных стекол. Такой вывод о поведении релаксационного вклада $G_{r}$ можно сделать как для исходного состояния, так для релаксированного. Однако, согласно табл. 1, составы на основе $\mathrm{Pd}$ и $\mathrm{Zr}$ принципиально отличаются между собой по ха- рактеру поведения нерелаксационной компоненты $G_{u r}$. Так, коэффициент $\alpha_{1}$ для стекол на основе $\mathrm{Pd}$ является положительным, в то время как для стекол на основе $\mathrm{Zr}$ - отрицательным. При этом коэффициент $\alpha_{2}$ по абсолютному значению примерно на порядок больше для палладиевых стекол. Другими словами, металлические стекла на основе Pd обладают принципиально разным ангармоническим вкладом по сравнению с циркониевыми стеклами, а их электронная подсистема оказывает значительно большее влияние на сдвиговую упругость некристаллической структуры.

Необходимо также обратить внимание на тот факт, что температура $T_{g}$ на термограммах ДСК определена по началу эндотермической реакции, т.е. когда начинает происходить переход «стекло - 
жидкость». Параллельно с эндотермическим эффектом наблюдается быстрое снижение величины $d G / d T$ (см. рис. 2). Такое поведение косвенным образом указывает на взаимосвязь активационных процессов теплопоглощения и релаксации модуля сдвига при переходе вещества из стеклообразного состояние в переохлажденное жидкое. Именно такая взаимосвязь тепловых эффектов и релаксации сдвиговой упругости металлических стекол была установлена в работах $[16,17]$.

\section{ЗАКЛЮЧЕНИЕ}

Выполнены измерения температурной зависимости модуля сдвига для металлических стекол $\mathrm{Pd}_{43.2} \mathrm{Cu}_{28} \mathrm{Ni}_{8.8} \mathrm{P}_{20}, \mathrm{Pd}_{41.25} \mathrm{Cu}_{41.25} \mathrm{P}_{17.5}, \mathrm{Zr}_{46} \mathrm{Cu}_{45} \mathrm{Al}_{7} \mathrm{Ti}_{2}$ и $\mathrm{Zr}_{46} \mathrm{Cu}_{46} \mathrm{Al}_{8}$ на частотах около $500 \mathrm{kHz}$. Измерения проведены, в том числе, при переходе вещества из твердого некристаллического состояния в переохлажденное жидкое. Установлено, что изменение мгновенного модуля сдвига некристаллической металлической среды при изменении температуры можно описать как сумму трех компонент - ангармонической, электронной и релаксационной. Первая компонента обусловлена ангармонизмом атомных колебаний, вторая - электронной подсистемой некристаллической структуры, а третья - процессами структурной релаксации. Установлено, что характер поведения релаксационной компоненты не зависит от химического состава металлических стекол, несмотря на разные их физические свойства, в том числе разный характер поведения ангармонической и электронной компонент. Релаксационная компонента растет ниже калориметрической температуры стеклования $T_{g}$, в то время как выше $T_{g}$ она снижается.

Полученные результаты свидетельствует о том, что механизм релаксации сдвиговой упругости металлических стекол является единообразным вне зависимости от химического состава.

\section{ИСТОЧНИК ФИНАНСИРОВАНИЯ}

Работа поддержана грантом Минобрнауки РФ № 3.1310.2017/4.6.

\section{БЛАГОДАРНОСТИ}

Автор выражает благодарность проф. В.А. Хонику за обсуждение статьи.

\section{КОНФЛИКТ ИНТЕРЕСОВ}

Авторы декларируют отсутствие явных и потенциальных конфликтов интересов, связанных с публикацией настоящей статьи.

\section{СПИСОК ЛИТЕРАТУРЫ}

1. Dyre J. C. // Reviews of Modern Physics, 2006, vol. 78, pp. 953-972. DOI: 10.1103/revmodphys.78.953

2. Dyre J. C., Olsen N. B., Christensen T. // Physical Review B, 1996, vol. 53, pp. 2171-2174. DOI: 10.1103/ physrevb.53.2171

3. Khonik V. A., Mitrofanov Yu. P., Lyakhov S. A., Vasiliev A. N., Khonik S. V., Khoviv D. A. // Physical Review B, 2009, vol. 79, pp. 132204-1-132204-4. DOI: 10.1103/ physrevb.79.132204

4. Chen H. S. // Reports on Progress in Physics, 1980, vol. 43, pp. 353-432. DOI: 10.1088/0034-4885/43/4/001

5. Hirao M., Ogi H. EMATS for Science and Industry: Noncontacting Ultrasonic Measurements. New-York, Springer, 2003, p. 372.

6. Васильев А. Н., Бучельников В. Д., Гуревич М. И., Каганов М. И., Гайдуков Ю. П. Электромагнитное возбуждение звука в металлах. Челябинск, Изд-во ЮУрГУ, 2001, 339 с.

7. Wang W. H. // Progress in Materials Science, 2012, vol. 57, pp. 487-656. DOI: 10.1016/j.pmatsci.2011.07.001

8. Watanabe L. Y., Roberts S. N., Baca N., Wiest A., Garrett S. J., Conner R. D. // Materials Science and Engineering: C, 2013, vol. 33, pp. 4021-4025. DOI: $10.1016 /$ j.msec.2013.05.044

9. Wang D. P., Zhao D. Q., Ding D. W., Bai H. Y., Wang W. H. // Journal of Applied Physics, 2014, vol. 115, pp. 123507-1-123507-4. DOI: 10.1063/1.4869548

10. Zhang Z., Keppens V., Liaw P. K., Yokoyama Y. // Journal of Materials Research, 2006, vol. 22, pp. 364-367. DOI: 10.1557/jmr.2007.0040

11. Хоник В. А. // Известия Академии наук. Серия физическая, 2001, т. 65, № 10, с. 1465-1471.

12. Штремель М. А. Прочность сплавов. Часть I. Дефекты решетки. М.: МИСИС, 1999, 384 с.

13. Gordon C. A., Granato A. V. // Materials Science and Engineering A, 2004, vol. 370, pp. 83-87. DOI: 10.1016/ j.msea.2003.08.077

14. Shen T. D., Schwarz R. B. // Applied Physics Letters, 2006, vol. 88, pp. 091903-1-091903-3. DOI: $10.1063 / 1.2172160$

15. Tsyplakov A. N., Mitrofanov Yu. P., Khonik V. A., Kobelev N. P., Kaloyan A. A. // Journal of Alloys and Compounds, 2015, vol. 618, pp. 449-454. DOI: 10.1016/j. jallcom.2014.08.198

16. Mitrofanov Y. P., Wang D. P., Makarov A. S., Wang W. H., Khonik V. A. // Scientific Reports, 2016, vol. 6, p. 23026-1-23026-6. DOI: 10.1038/srep23026

17. Afonin G. V., Mitrofanov Yu. P., Makarov A. S., Kobelev N. P., Khonik V. A. // Journal of Non-Crystalline Solids, 2017, vol. 475, pp. 48-52. DOI: 10.1016/j. jnoncrysol.2017.08.029 
UDC 538.951, 539.3, 539.5

DOI: $10.17308 / \mathrm{kcmf} .2019 .21 / 719$

Received 13.01.2019

Accepted 15.02.2019

\title{
FEATURES OF THE SHEAR ELASTICITY RELAXATION OF METALLIC GLASSES
}

\author{
(C) 2019 Yu. P. Mitrofanov* \\ Voronezh State Pedagogical University \\ 86, Lenina str., 394043 Voronezh, Russian Federation
}

\begin{abstract}
Purpose. The work is aimed at a determination of the regularities of the changes of the shear elasticity occurring upon structural relaxation of metallic glasses.

Methods and methodology. Glassy $\mathrm{Pd}_{43.2} \mathrm{Cu}_{28} \mathrm{Ni}_{8.8} \mathrm{P}_{20}, \mathrm{Pd}_{41.25} \mathrm{Cu}_{41.25} \mathrm{P}_{17.5}, \mathrm{Zr}_{46} \mathrm{Cu}_{46} \mathrm{Al}_{8}$ and $\mathrm{Zr}_{46} \mathrm{Cu}_{45} \mathrm{Al}_{7} \mathrm{Ti}_{2}$ (at. \%) produced by melt suction (Zr-based MGs) and melt jet quenching (Pd-based MGs) were chosen for the investigation. In situ measurements of the shear modulus $G$ were carried out at frequencies of about $500 \mathrm{kHz}$ at a high relative precision of up to $\approx 5 \mathrm{ppm}$ by the electromagnetic acoustic resonance method.

Results. The change of modulus $G$ with temperature can be described as the sum of three components anharmonic, electronic and relaxation. Despite the differences in the physical properties of metallic glasses under investigations (chemical composition, glass forming ability, glass transition temperatures, etc.) including the different behavior of the anharmonic and electronic components, one observes distinctive common regularities of the relaxation of their shear elasticity upon heat treatment. It is found that structural relaxation leads to an increase of shear modulus below the glass transition temperature $T_{g}$ and decreases it at $T>T_{g}$.

Conclusions. It is concluded that the mechanism of shear elasticity relaxation of non-crystalline metallic structures is universal despite of variations of the chemical composition.
\end{abstract}

Keywords: metallic glasses, shear elasticity, relaxation.

\section{SOURCE OF FINANCING}

This work was supported by the Ministry of Education and Science of the Russian Federation (grant No. 3.1310.2017 / 4.6).

\section{CONFLICT OF INTEREST}

The authors declare the absence of obvious and potential conflicts of interest related to the publication of this article.

\section{ACKNOWLEDGMENTS}

The author thanks prof. V.A. Khonik for discussing the article.

\section{REFERENCES}

1. Dyre J. C. Reviews of Modern Physics, 2006, vol. 78, pp. 953-972. DOI: 10.1103/revmodphys.78.953

2. Dyre J. C., Olsen N. B., Christensen T. Physical Review B, 1996, vol. 53, pp. 2171-2174. DOI: 10.1103/ physrevb.53.2171

3. Khonik V. A., Mitrofanov Yu. P., Lyakhov S. A., Vasiliev A. N., Khonik S. V., Khoviv D. A. Physical Review

$\triangle$ Mitrofanov Yuriy P., e-mail: mitrofanovyup@gmail.com.
B, 2009, vol. 79, pp. 132204-1-132204-4. DOI: 10.1103/ physrevb.79.132204

4. Chen H. S. Reports on Progress in Physics, 1980, vol. 43, pp. 353-432. DOI: 10.1088/0034-4885/43/4/001

5. Hirao M., Ogi H. EMATS for science and industry: noncontacting ultrasonic measurements. New-York, Springer, 2003, p. 372.

6. Vasil'ev A. N., Buchel'nikov V. D., Gurevich M. I., Kaganov M. I., Gajdukov Ju. P. Electromagnetic Excitation of Sound in Metals. Cheljabinsk, Izd-vo JuUrGU Publ., 2001, 339 p.

7. Wang W. H. Progress in Materials Science, 2012, vol. 57, pp. 487-656. DOI: 10.1016/j.pmatsci.2011.07.001

8. Watanabe L. Y., Roberts S. N., Baca N., Wiest A., Garrett S. J., Conner R. D. Materials Science and Engineering: C, 2013, vol. 33, pp. 4021-4025. DOI: 10.1016/j.msec.2013.05.044

9. Wang D. P., Zhao D. Q., Ding D. W., Bai H. Y., Wang W. H. Journal of Applied Physics, 2014, vol. 115, pp. 123507-1-123507-4. DOI: 10.1063/1.4869548

10. Zhang Z., Keppens V., Liaw P. K., Yokoyama Y. Journal of Materials Research, 2006, vol. 22, pp. 364-367. DOI: $10.1557 /$ jmr.2007.0040 
11. Khonik V. A. Izvestija Akademii Nauk. Serija fizicheskaja [Bulletin of the Russian Academy of Sciences: Physics], 2001, vol. 65, no. 10, pp. 1465-1471. (in Russ.)

12. Shtremel' M. A. The Strength of the Alloys. Part I. Defects of the Lattice. Moscow, MISIS Publ., 1999, 384 p. (in Russ.)

13. Gordon C. A., Granato A. V. Materials Science and Engineering A, 2004, vol. 370, pp. 83-87. DOI: 10.1016/j. msea.2003.08.077

14. Shen T. D., Schwarz R. B. Applied Physics Letters, 2006, vol. 88, pp. 091903-1-091903-3. DOI: $10.1063 / 1.2172160$
15. Tsyplakov A. N., Mitrofanov Yu. P., Khonik V. A., Kobelev N. P., Kaloyan A. A. Journal of Alloys and Compounds, 2015, vol. 618, pp. 449-454. DOI: 10.1016/j. jallcom.2014.08.198

16. Mitrofanov Y. P., Wang D. P., Makarov A. S., Wang W. H., Khonik V. A. // Scientific Reports, 2016, vol. 6, p. 23026-1-23026-6. DOI: 10.1038/srep23026

17. Afonin G. V., Mitrofanov Yu. P., Makarov A. S., Kobelev N. P., Khonik V. A. // Journal of Non-Crystalline Solids, 2017, vol. 475, pp. 48-52. DOI: 10.1016/j. jnoncrysol.2017.08.029
Юрий Петрович Митрофанов - к. ф.-м. н., доцент, Воронежский государственный педагогический университет, Воронеж, Российская Федерация; e-mail: mitrofanovyup@gmail.com. ORCID iD 00000002-7939-5230.
Mitrofanov Yuriy P. - Cand. Sci. (Phys.-Math.), Assistant Professor, Voronezh State Pedagogical University, Voronezh, Russian Federation; e-mail: mitrofanovyup@gmail.com. ORCID iD 0000-0002-79395230. 